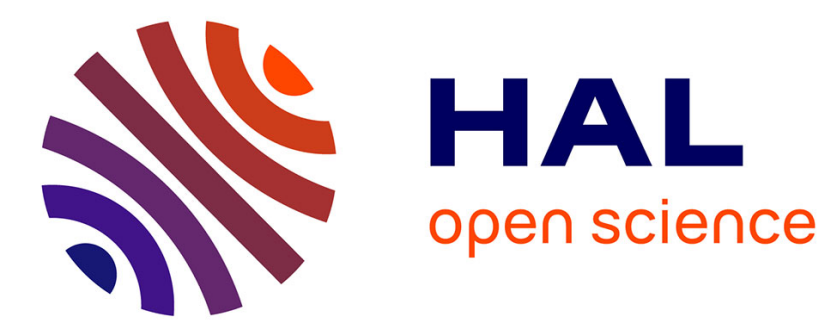

\title{
BANDWIDTH ENLARGEMENT OF PLANAR EBG ANTENNAS
}

Thai Hung Vu, Kouroch Mahdjoubi, Anne-Claude Tarot, Sylvain Collardey

\section{To cite this version:}

Thai Hung Vu, Kouroch Mahdjoubi, Anne-Claude Tarot, Sylvain Collardey. BANDWIDTH ENLARGEMENT OF PLANAR EBG ANTENNAS. LAPC 2007 (Loughborough Antennas \& Propagation Conference), Apr 2007, Loughborough, United Kingdom. pp.152-153. hal-00142219

\section{HAL Id: hal-00142219 https://hal.science/hal-00142219}

Submitted on 17 Apr 2007

HAL is a multi-disciplinary open access archive for the deposit and dissemination of scientific research documents, whether they are published or not. The documents may come from teaching and research institutions in France or abroad, or from public or private research centers.
L'archive ouverte pluridisciplinaire HAL, est destinée au dépôt et à la diffusion de documents scientifiques de niveau recherche, publiés ou non, émanant des établissements d'enseignement et de recherche français ou étrangers, des laboratoires publics ou privés. 


\title{
BANDWIDTH ENLARGEMENT OF PLANAR EBG ANTENNAS
}

\author{
Thai-Hung VU, Kourosh MAHDJOUBI, Anne-Claude TAROT, Sylvain COLLARDEY
IETR, UMR CNRS 6164, Université de Rennes 1, Campus de Beaulieu, 35042 Rennes Cedex, France

\author{
thai-hung.vu@univ-rennes1.fr, mahdjoubi@univ-rennes1.fr
}

\begin{abstract}
A new structure based on a combination of two PRS (Partially Reflective Surface) is introduced and studied analytically to improve the bandwidth of the FP and EBG antennas. The original procedure developed here leads to a design method for broadening the bandwidth of planar EBG antennas.

\section{Introduction}

EBG antennas are generally composed of an EBG material (Electromagnetic Band Gap), a reflector plane and a primary source that is placed inside the structure (Fig. 1). These antennas are capable to produce highly directive and narrow beam, while they are very compact and low profile, compared to classical directive antennas (horn, parabolic reflector, etc), but it usually presents a very narrow bandwidth (about $0 . \% 1-1 \%$ ). In this poster, we will first remind the principles of the EBG antennas, explain the reason why they usually have a narrow bandwidth and the methods to improve it. We will after introduce a new structure based on a combination of two PRS which enables us to enlarge the bandwidth of the traditional EBG antennas. We also present a simple analytical method, based on the input impedance of a plane wave source placed inside the structure, to evaluate the bandwidth of the EBG antennas.
\end{abstract}

\section{I -Principles of the Fabry-Perot (FP) and EBG antennas}

Let us consider a plane wave source inside a Fabry-Perot cavity (Fig. 1). The cavity is made of two infinite Partially Reflective Surfaces (PRS) characterized by their transmission $\left(t_{1} ; t_{2}\right)$ and reflexion coefficients $\left(r_{1} ; r_{2}\right)$. By applying the method of the successive reflections inside the cavity, the total transmitted wave T (i.e. the FP response) can be given as a superposition of all partially transmitted fields and is shown in (Eq. 1).

$$
\begin{gathered}
T_{F P}(\theta)=t_{1}\left(1+\sum_{n=1}^{\infty}\left(r_{1} r_{2}\right)^{n} \exp (-2 j k n D \cos (\theta))+\sum_{n=1}^{\infty} r_{2}\left(r_{1} r_{2}\right)^{n} \exp \left(-2 j k n D \cos (\theta)-2 j k D_{2} \cos (\theta)\right)\right) \\
T_{F P}(\theta)=\frac{t_{1}\left(1+r_{2} \exp \left(-2 j k D_{2} \cos (\theta)\right)\right)}{1-r_{1} r_{2} \exp (-2 j k D \cos (\theta))}
\end{gathered}
$$

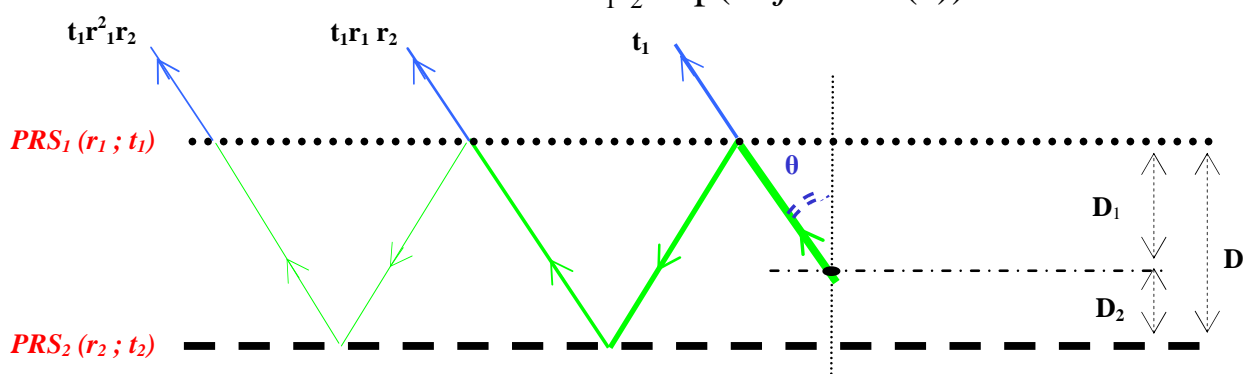

Fig. 1 : Method of the successive reflections for studying the EBG antennas

Maximum of the directivity is reached at the frequency where the module of the coefficient of transmission is maximal, or the denominator in Eq. 1 is minimal. At this frequency and for the normal incidence $(\theta=0)$, we have

$$
\varphi_{r 1}+\varphi_{r 2}=2 k D
$$

$\varphi_{\mathrm{r} 1}$ and $\varphi_{\mathrm{r} 2}$ are the phase of the two PRS at this frequency, consecutively. 
Usually, the left hand member of the (Eq. 2) $\varphi(f)=\varphi_{\mathrm{r} 1}+\varphi_{\mathrm{r} 2}$ is a decreasing function versus frequency and the right hand member $\mathrm{F}(\mathrm{f})=2 \mathrm{kD}=4 \pi \mathrm{Df} / \mathrm{c}$ an increasing function. Therefore, the Eq. 2 is satisfied at only one frequency (Fig. 2a). Let us define "out-of-phase" tolerances $\Delta \varphi_{1}$ and $\Delta \varphi_{2}$ for Eq. 2 as the phase difference $\Delta \varphi_{1}=\left|F\left(f_{1}\right)-\varphi\left(f_{1}\right)\right|$ and $\Delta \varphi_{2}=\left|F\left(f_{2}\right)-\varphi\left(f_{2}\right)\right|$ (see Fig. 2). The frequency bandwidth thus becomes: $\Delta f=f_{2}-f_{1}$. From Fig. $2 \mathrm{a}$, one can see that this kind of antenna has an extremely narrow bandwidth $(0.1-1 \%)$. There are two methods to increase the bandwidth: either to reduce the slope of the curves $\varphi$ (f) and F(f) (Fig. 2b) [2], or to invert the $\varphi(f)$ slope (Fig. 2c). The second method may require many simulations and computation time [3]. In this paper, we propose a fast analytical method to achieve the slope inversion.

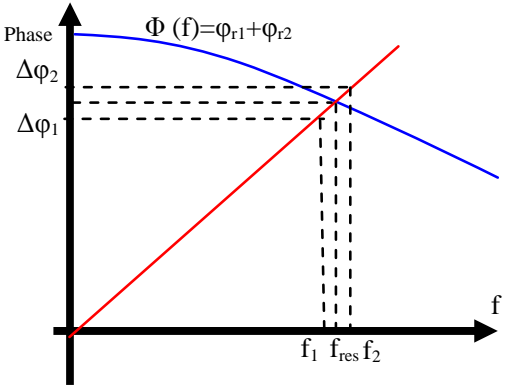

(a)

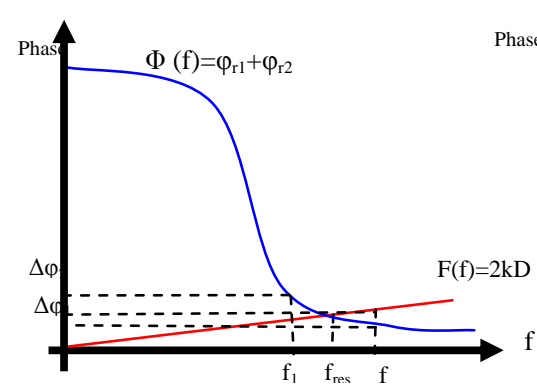

(b)

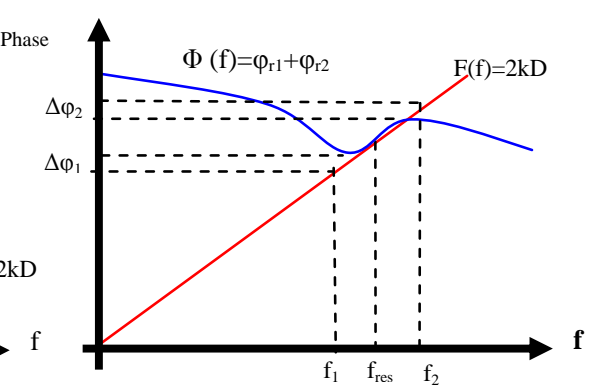

(c)

Fig. 2 Classical EBG antenna (a) and different methods for enlarging the bandwidth of the EBG antennas :

(b): reduced slope $\varphi(f),(c)$ : inverted slope $\varphi(f)$

\section{Combined Partially Reflective Surface}

Let us replace the $\mathrm{PRS}_{1}$ of the Fig.1 by a new structure composed of two different PRS (PRS $\left.1 \mathrm{a}, \mathrm{PRS}_{1 \mathrm{~b}}\right)$, separated from each other by a distance $d$. The structure is excited by an incident plane wave placed outside (Fig. 3).

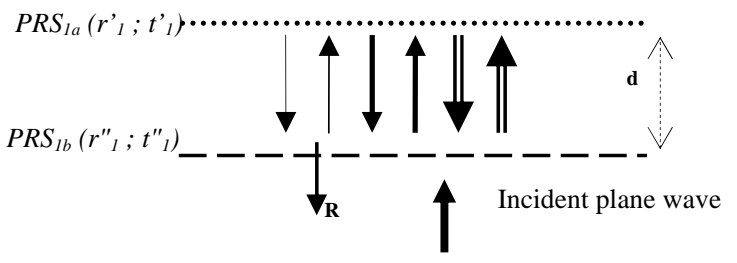

Fig. 3 : New PRS 1 composed of two sub-PRS $\left(\mathrm{PRS}_{1 \mathrm{a}} \& \mathrm{PRS}_{1 \mathrm{~b}}\right)$

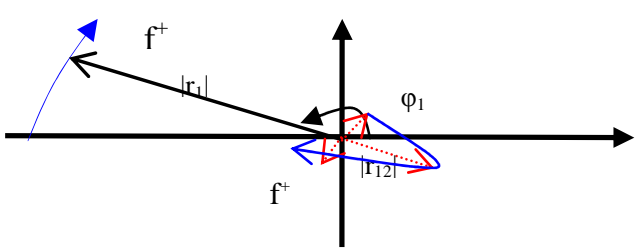

Fig. 4: $r_{1}{ }_{1} \& r_{12}$ in polar coordinate

The reflexion coefficient $R$ of the new PRS can be calculated by applying the same method as used in (Fig.1):

$$
R=r_{1}^{\prime}+\frac{t_{1}^{\prime 2} r_{1}^{\prime \prime} \exp (-2 j k d)}{1-r_{1}^{\prime} r_{1}^{\prime \prime} \exp (-2 j k d)}=r_{1}^{\prime}+r_{12}
$$

At the resonance frequency, the module of $R$ is minimal. This means that the module of $r_{12}$ reaches (nearly) its maximum and $r^{\prime}{ }_{1}$ and $r_{12}$ become out of phase:

$$
\varphi_{r^{\prime} 1}=\varphi_{r 12}+\pi
$$

For a given $r_{1}{ }_{l}$ and $r{ }_{1}$ we can adjust the parameter $d$ so that Eq. 4 is satisfied.

If $\left|\mathrm{r}_{1}{ }_{1}\right|>\left|\mathrm{r}^{\prime}{ }_{1}\right|$, we always have $\left|\mathrm{r}^{\prime}{ }_{1}\right|>\left|\mathrm{r}_{12}\right|$ because:

$$
\left|\frac{r_{1}^{\prime}}{r_{12}}\right| \geq \frac{\left|r_{1}^{\prime}\right|}{\left|t_{1}^{\prime 2} r_{1}^{\prime \prime}\right| /\left(1-\left|r_{1}^{\prime} r_{1}^{\prime \prime}\right|\right)}=\frac{\left|r_{1}^{\prime}\right|\left(1-\left|r_{1}^{\prime} r_{1}^{\prime \prime}\right|\right)}{\left|r_{1}^{\prime \prime}\right|\left(1-\left|r_{1}^{\prime 2}\right|\right)}=\frac{\left|r_{1}^{\prime}\right|-\left|r_{1}^{\prime 2} r_{1}^{\prime \prime}\right|}{\left|r_{1}^{\prime \prime}\right|-\left|r_{1}^{\prime 2} r_{1}^{\prime \prime}\right|} \geq 1
$$

In this case, the phase of $R$ follows in general that of $r_{1}{ }_{1}$ except at the resonance frequency, where $\left|r_{12}\right|$ becomes comparable to $\left|r_{l}{ }_{1}\right|$. At this frequency, phase of $R$ is subjected to an important disturbance (Fig. 5) before it turns back to that of $r_{1}{ }_{1}$. With a good choice of the two PRS, It's possible to inverse the slope of the phase of R (Eq. 3) around this frequency. 
In Fig. 5 and Fig. 6 we give an example of a combined surface $\mathrm{PRS}_{1}$ made by two PRS constituted of metallic strips (PRS $1 \mathrm{a}: a / P t=20 \%, P t=10 \mathrm{~mm}, \mathrm{PRS}_{1 \mathrm{~b}}: a / P t=5 \%, P t=20 \mathrm{~mm}$, a: strip width, Pt: period of strip). The distance $d$ between the two PRS is chosen $(d=54.3275 \mathrm{~mm})$ so that the combined PRS $_{1}$ has a resonance frequency at 2.4 $\mathrm{GHz}$. We can see that an inversion in the phase slope of $R$ is obtained from (nearly) $2.3 \mathrm{GHz}$ to $2.5 \mathrm{GHz}$.

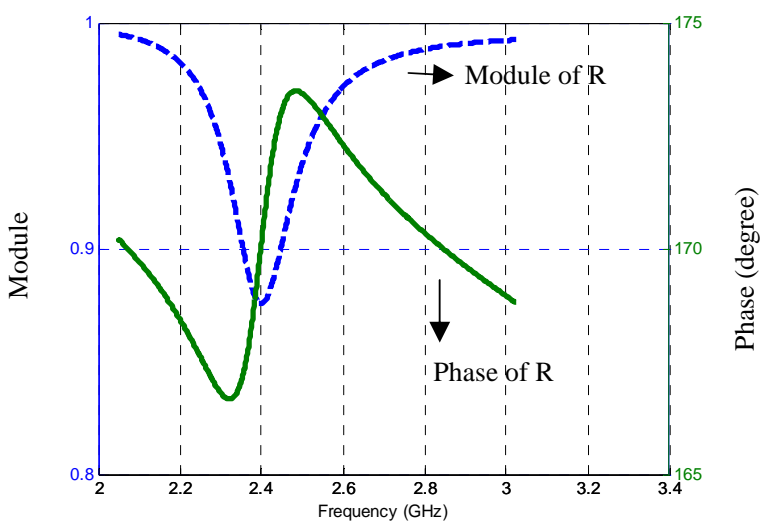

Fig. 5: Example of a combined PRS which gives an increase in phase for the reflection coefficient

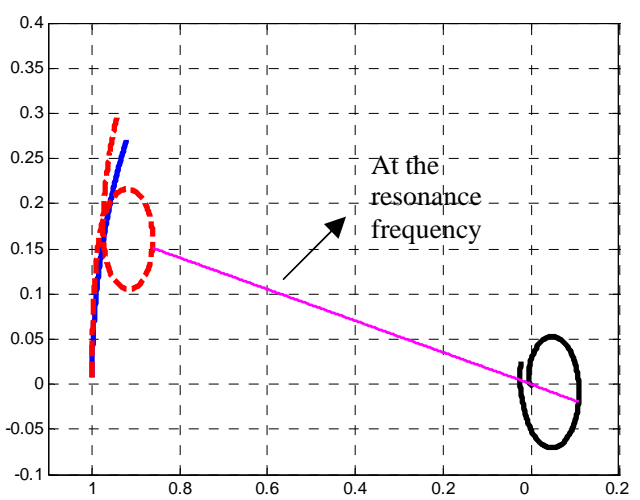

Fig. 6: on the left: $r^{\prime}{ }_{1}$ (solid-curve), $R$ (dashedcurve). On the right: $r_{12}$ (solid-curve) of the combined PRS in polar coordinate

In the following section, we will show that this type of surface enables us to improve the radiation bandwidth of EBG antennas. We will also present a simple analytical method, based on the input impedance of a plane wave source placed inside the cavity, which can be used to evaluate the impedance bandwidth of the EBG antennas.

\section{Bandwidth for input impedance and radiation pattern of the exciting plane wave}

Let us consider an exciting plane wave source with normal incident placed inside the FP cavity. The input impedance of the source (the ratio between the E field and $\mathrm{H}$ field at the source) can be written as [4]:

$$
Z=\frac{Z_{1} Z_{2}}{Z_{1}+Z_{2}}
$$

where

$$
Z_{1}=\frac{E_{z}^{\text {sup }}}{H_{y}^{\text {sup }}}=\eta \frac{\left(1+r_{2} \exp \left(-2 j k D_{2}\right)\right)}{\left(1-r_{2} \exp \left(-2 j k D_{2}\right)\right)} \quad Z_{2}=\frac{E_{z}^{\text {sup }}}{H_{y}^{\text {sup }}}=\eta \frac{\left(1+r_{1} \exp \left(-2 j k D_{1}\right)\right)}{\left(1-r_{1} \exp \left(-2 j k D_{1}\right)\right)}
$$

$\eta$ is the free space intrinsic impedance.

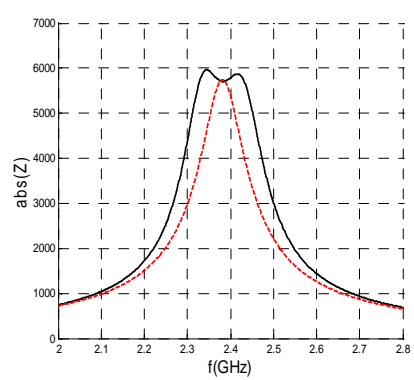

Fig. 7: Comparison of the impedance modules
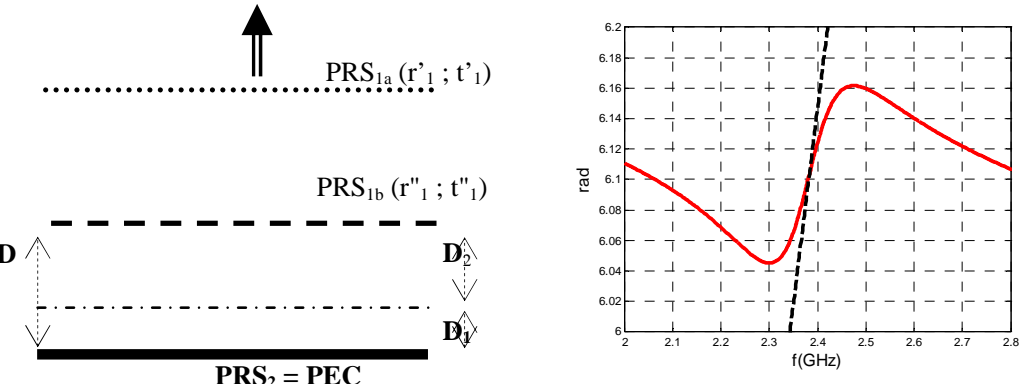

Fig. 8: Structure PEC + combined PRS (left) and its phase (right)

Fig. 7 shows the impedance module calculated from Eq. 5. The dashed curve represents the impedance of the traditional cavity composed of a simple PRS and a PEC (perfect magnetic conductor). The solid curve represents the impedance of the new cavity composed of a combined PRS and a PEC (see Fig.8). It is worth to note that at the resonance frequency, which is the same for the two cavities $(\mathrm{f}=2.3834 \mathrm{GHz})$, the two PRS also have a same reflection coefficient $\left(\mathrm{r}=0.8787 / 168.86^{\circ}\right)$. The plane wave source is placed in the middle of the cavity $\left(\mathrm{D}_{1}=\mathrm{D}_{2}\right)$ in the both cases. 
We can notice that the plane wave input impedance of the new cavity (with combined PRS) has a larger bandwidth the traditional cavity as it was expected.

Fig.9 shows the radiation pattern (Eq. 1) of the two structures at different frequencies around the resonance frequency. The variation in the level and in the form of the pattern for the new antenna (dashed curves) is much less than that for the traditional one. This confirms the bandwidth enlargement for the radiation pattern well as.

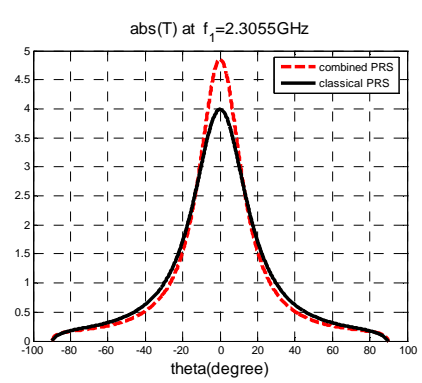

(a) $\mathrm{F}_{1}=2,305 \mathrm{GHz}$

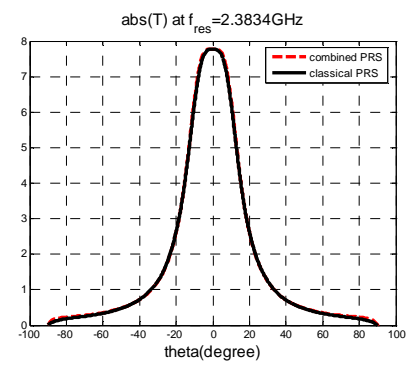

(c) $\mathrm{F}_{\text {res }}=2,383 \mathrm{GHz}$

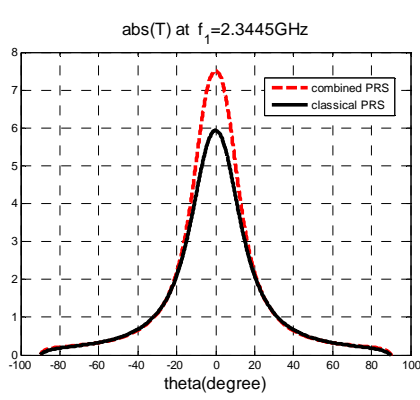

(b) $\mathrm{F}_{1}=2,344 \mathrm{GHz}$

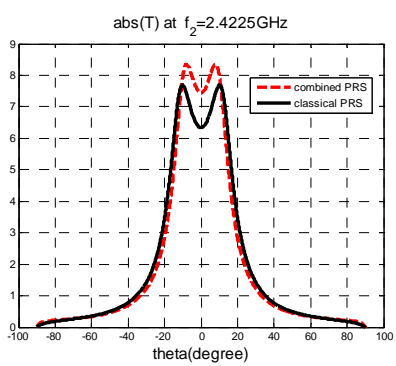

(d) $\mathrm{F}_{2}=2,422 \mathrm{GHz}$

Fig. 9: Comparison of the radiation patterns at different frequencies

\section{Conclusions}

A new design method and analytical expression are proposed to enlarge the bandwidth of Fabry-Perot antennas. The design which is based on the combination of two PRS (Partially Reflective Surface), can be easily generalised to the treatment of EBG (multilayer) antennas using a simple recursive method [5]. The bandwidth improvement has been verified on the radiation characteristics and the input impedance of the new antenna. We are currently working on the application of more than two PRS, on the combination of the techniques of the (Fig. 2b) (PMC reflectors) and (Fig. 2c) (combined PRS) and also on the optimisation of the EBG parameters to attain the maximum improvement of the bandwidth or to achieve multi-criterion objectives (bandwidth, beamwidth, side lobes level, etc.).

\section{References}

[1] G.V. Trentini, "Partially reflecting sheet arrays", IRE Trans. On Antenna and Propagation, 1956, vol. 4, pp. 666-671.

[2] A. Ourir, A. de Lustrac, and Jean-Michel Lourtioz, "All-metamaterial-based subwavelength cavities $(\lambda / 60)$ for ultrathin directive antennas", Applied Physic Letters, No 88, 084103, 2006

[3] H. P. Feresidis, J. C. Vardaxoglou, “A broadband high-gain resonant cavity antenna with single feed", Pro. 'EuroCAP 2006', Nice, France 2006.

[4] T.H. VU, Master thesis, IETR, University of Rennes I, France, June 2005.

[5] H. Boutayeb, K. Mahdjoubi and A.C Tarot, "Multi-layer crystals of metallic wires: Analysis of the transmission coefficient for outside and inside excitation", Progress In Electromagnetics Research, PIER 59, pp. 299-324, 2006. 